\title{
LA PERCEPCIÓN DE RIESGO EN ESTUDIANTES UNIVERSITARIOS ANTE LA PROPAGACIÓN DEL CORONAVIRUS SARS-COV-2 Y LA ENFERMEDAD COVID-19
}

THE PERCEPTION OF RISK IN UNIVERSITY STUDENTS BEFORE THE SPREAD OF THE SARSCOV-2 CORONAVIRUS AND THE COVID-19 DISEASE

\author{
Margarita Juárez-Nájera*, José Marcos Bustos-Aguayo**, \\ Javier Carreón Guillén**, Cruz García-Lirios* \\ *Universidad Autónoma Metropolitana, **Universidad Nacional Autónoma de México, México \\ Correspondencia: garcialirios@yahoo.com
}

\section{RESUMEN}

El objetivo del presente documento fue explorar la confiabilidad y validez de un instrumento que mide la percepción de riesgo en estudiantes de licenciatura respecto a la propagación del coronavirus covid-19. Se realizó un trabajo transversal y correlacional con una selección de 100 estudiantes de una universidad pública, considerando su participación en las prácticas profesionales y servicio social en centros de salud comunitaria del centro de México. Se observaron tres dimensiones relativas a la inconmensurabilidad, impredecibilidad e incontrolabilidad del riesgo que explicaron el $77 \%$ de la varianza, aunque el diseño de la investigación limitó los resultados a la muestra encuestada, se sugiere la extensión del estudio a otras entidades del servicio de salud pública.

Palabras clave: Seguridad, riesgo, propagación, contagio, coronavirus, covid-19

\section{ABSTRACT}

The objective of this documççent was to explore the reliability and validity of an instrument that measures the perception of risk in undergraduate students regarding the spread of covid-19 coronavirus. A cross-sectional and correlational work was carried out with a selection of 100 students from a public university, considering their participation in 
professional practices and social service in community health centers in central Mexico. Three dimensions were observed regarding the incommensurability, unpredictability and uncontrollability of the risk that explained $77 \%$ of the variance, although the research design limited the results to the sample surveyed, the extension of the study to other health service public entities is suggested.

Key words: Security, risk, spread, contagion, coronavirus, covid-19

\section{INTRODUCCIÓN}

Hasta el momento en que se escribe este documento, la política de mitigación de la pandemia ocasionada por el coronavirus SARS-COV-2 y el confinamiento de las personas para reducir los contagios y la enfermedad COVID-19 se llevan a cabo debido a su alta taza de transmisibilidad en personas y letalidad en adultos mayores (OMS, 2020). Debido a que algunos síntomas como fiebre, tos seca y cansancio son indicadores del ataque del coronavirus al sistema inmunológico, la comunidad científica asume que quienes los presentan deben realizarse pruebas confirmatorias que oscilan entre dos horas y cuatro días (OPS, 2020). En la medida en que se documentan más casos, otros síntomas están asociados como diarrea, conjuntivitis, dolor en garganta y cabeza, pérdida de los sentidos del gusto y olfato, así como erupciones y pérdida de color en dedos, manos y pies.

Sin embargo, la comunidad científica parece coincidir en la observación de síntomas denominados como graves; sensación de falta de aire, dificultad al respirar, dolor o presión en el pecho e incapacidad para hablar y moverse ameritan cuidados intensivos y respiración asistida por ventilación (OMS, 2020). En ese sentido, a escala mundial se contabilizan 4 millones de infectados, un millón 350 recuperados y 300 mil muertes confirmados junto a otro número equivalente de víctimas por confirmar (OPS, 2020). En México, la medición ha sido reconocida por el gobierno como inconsistente debido a los métodos y técnicas empleados, aunque se reconocen poco más de 31 mil infectados, casi 18 mil recuperados y 3160 defunciones hasta la primera semana de mayo de 2020 (SSA, 2020).

En el marco de la propagación de enfermedades, los estudios sobre el riesgo al contagio, principalmente los relativos a la intervención social, sectorial, gremial, institucional, laboral, barrial o individual han demostrado la emergencia de una agenda centrada en ejes y temas de discusión relativos a los riesgos a la salud (Aldana, 2018). 
En el caso de una probable propagación del coronavirus covid-19, la edificación de una agenda se gesta desde la difusión de los casos, aún y cuando estos se perciban distantes, las audiencias buscan información a fin de poder difundirla o compararla para reprocesarla como un riesgo social, local y personal (Bustos, 2018).

De este modo es que el estudio de la propagación del coronavirus covid-19 desde las expectativas de opiniones contribuirá a la discusión en torno a las políticas de mitigación o contención de las relaciones entre personas con el fin de evitar contagios, explicar los escenarios de riesgo y anticipar las amenazas potenciales a la salud de sectores instituciones, grupos y personas (Garcia, 2020).

En el presente trabajo se revisan los marcos teóricos, conceptuales y empíricos de los riesgos percibidos ante la propagación de enfermedades. Una vez establecidos los ejes de discusión, se exponen los hallazgos principales, relacionándolos con los planteamientos teóricos que dan cuenta del fenómeno al establecer la observación y medición sistemática de un fenómeno emergente y por tanto explorable en su estructura como lo es la percepción del riesgo al contagio del coronavirus covid-19.

\section{TEORÍA DE LA PERCEPCIÓN DE RIESGOS}

En las ciencias sociales los fenómenos emergentes, inciertos y amenazantes como la propagación del coronavirus covid-19 se explican desde la teoría de la percepción del riesgo. Se trata de una serie de planteamientos, algunos de ellos observados sistemáticamente en diferentes sectores poblacionales como los grupos vulnerables de adultos mayores, en torno al efecto de la información sobre un fenómeno desconocido (Carreón, 2017).

La raíz epistemológica de la percepción del riesgo está sustentada en la fenomenología social que centra su atención en la epojé o descripción de una serie de indicadores convergentes en la inconmensurabilidad, impredecibilidad e incontrolabilidad de eventos considerados amenazas por su desconocimiento (Carreón, 2018).

Sobre la base de la epojé o suspensión de expectativas, la teoría de la percepción de riesgo considera que la racionalidad no es exclusiva o sustituida por la emotividad que supone un evento esperado de alto impacto y consecuencias a la integridad o salud de las personas (García, 2016). Es el caso de las decisiones prospectivas que destacan la incidencia de heurísticos o intuiciones racionales y emocionales sobre el comportamiento (Juarez, 2020). 
Esa epojé sugiere que si bien son emocionales los primeros síntomas de las personas al presenciar o enterarse de un evento descomunal como el covid-19 subyace una cuasi racionalidad que pretende calcular el fenómeno, anticipar sus efectos y llevar a cabo acciones preventivas o de contención ante la propagación inminente del coronavirus (García, 2017).

De este modo, la teoría de la percepción de riesgos se ha edificado a partir de estudios que observan sistemáticamente la epojé o suspensión emocional y racional de quienes son víctimas potenciales, espectadores mediáticos o futuros implicados en una propagación de enfermedades (Juárez, 2017).

Sin embargo, la teoría ha transitado de una exploración y descripción de las partes interesadas en un evento de riesgo a la explicación de sus comportamientos o la comprensión de sus actos en situaciones límite que obligan a las personas a suspender sus planes de trabajo, familiares o recreativos a fin de poder procesar la información concerniente a la problemática (Martínez, 2018).

Antes de este proceso, las audiencias de los medios de comunicación tradicionales y las redes sociales difunden información del evento, resaltando las amenazas y las víctimas potenciales (Mejía, 2016). En ese marco de especulación subyace la percepción del riesgo y los estudios correspondientes dan cuenta de este fenómeno tridimensional (Garcia, 2019).

\section{ESTUDIOS DE LA PERCEPCIÓN DE RIESGOS}

Las investigaciones pioneras de la percepción del riesgo observaron un cúmulo de emociones asociadas a los eventos, los procesos y las situaciones parámetros de tolerancia o permisibilidad para establecer umbrales de expectativas de cambio inminente desfavorable (Mendoza, 2017). En esos trabajos clásicos la propagación de enfermedades se midió desde la interrelación de variables como la exposición a eventos, la extensión de espacios, la capacidad de respuesta institucional, organizacional o civil (Quintero, 2019).

El desarrollo científico y tecnológico agregó nuevas variables a la ecuación. Son los casos de la influencia de los medios de comunicación y el establecimiento de una agenda pública en torno a la que se organizaron sectores públicos y sociales, así como actores políticos y civiles ante una crisis inminente (Quintero, 2017). El efecto de la difusión mediática en opiniones de las audiencias fue una fase central en los estudios de la percepción de riesgos ya que significó el abordaje de la dimensión emocional 
En su fase histórica emocional, los estudios de la percepción del riesgo establecieron un continuum que va del miedo, el enojo, la zozobra a la desesperanza aprendida para explicar la exposición a riesgos, aun cuando la difusión se intensifique (Rincón, 2018). A diferencia de las investigaciones clásicas, la percepción del riesgo se consideró como un componente resultante de la coexistencia de emociones.

Si los estudios pioneros destacaban la pasividad de víctimas potenciales para resaltar la intervención del Estado, los estudios emocionales reconocieron la prevención de riesgos a partir del control emocional de las potenciales víctimas en función de la difusión mediática. La contribución de los trabajos clásicos, mediáticos y emocionales a los estudios de los riesgos ha sido sustancial para establecer zonas vulnerables, eventos potenciales, situaciones probables o la formación de un capital humano para su afrontamiento.

Precisamente, las investigaciones contemporáneas de la percepción del riesgo no sólo recuperan los aportes de los estudios clásicos, mediáticos y emocionales, además plantean la prevalencia de tres dimensiones relativas a la evaluación, predicción y formación de capital humano para prevenir, afrontar y reducir riesgos.

En la dimensión evaluativa el reconocimiento de los límites científicos, tecnológicos, económicos, políticos y sociales configura la agenda preventiva y reactiva ante los riesgos potenciales, aunque prevalece el debate en torno a la percepción o expectativa de riesgos como una resultante emocional, racional o híbrida.

\section{MODELO DEL RIESGO ESPERADO RESPECTO A LA PROPAGACIÓN DEL CORONAVIRUS COVID-19}

A partir de los marcos teóricos, conceptuales y empíricos de la percepción del riesgo es posible delinear los ejes, trayectorias y relaciones entre las dimensiones de inconmensurabilidad, impredecibilidad e incontrolabilidad de la propagación del coronavirus covid-19.

La teoría de la percepción del riesgo ha establecido que la propagación de enfermedades supone efectos significativamente emocionales en la población, aunque también advierte la emergencia de la desconfianza hacia el Estado, principalmente las instituciones de salud pública para la comunicación y el manejo de los contagios, el aislamiento y la mitigación de casos (Hernandez, 2020). 
Aunada a esa desconfianza estatal e institucional, sectores instruidos de la sociedad civil cuestionan los recursos y las capacidades gubernamentales y sanitarias para afrontar la crisis, reconociendo la imposibilidad financiera, científica y tecnológica para la solución a la problemática (Limon, 2020).

Ambos fenómenos, la desconfianza estatal institucional y científica tecnológica son percepciones y expectativas de riesgo en tanto que emergen como respuesta común ante un brote de coronavirus. En el primer caso, los sectores públicos y actores políticos se han ganado la desconfianza ciudadana por su pasividad, negligencia, manipulación u opacidad informativa. En el segundo aspecto, derivado de la desconfianza hacia los políticos, administradores y servidores públicos, la sociedad civil reconoce que el financiamiento público a la ciencia y tecnología aplicada a la salud esta reducida a su mínima expresión.

Además, este escenario se recrudece si se considera que un proceso psicológico básico de las audiencias y víctimas potenciales de un evento de riesgo desarrollan una desesperanza aprendida a partir de la desconfianza política. Sumadas estas variables; desconfianza política y desesperanza aprendida, es posible observar que sus indicadores de inconmensurabilidad, impredecibilidad e incontrolabilidad se incrementan a partir de la difusión mediática de casos de contagio y muertes por el coronavirus covid- 19 .

De este modo es que un modelo explicativo de la percepción de riesgos ante la propagación del coronavirus covid-19 incluye las tres dimensiones en función del establecimiento de una agenda informativa de casos, aunque la experiencia de los síntomas exacerba las expectativas de riesgo.

Por consiguiente, el objetivo del presente trabajo fue comparar las dimensiones teóricas, conceptuales y empíricas con las dimensiones observadas a fin de poder discutir la fiabilidad y la validez del instrumento que mide la percepción de riesgo ante la propagación del virus covid-19

Formulación. ¿Existen diferencias significativas entre las dimensiones perceptuales de riesgo publicadas en la literatura con respecto a las dimensiones observadas en un estudio con estudiantes de una universidad pública del centro de México? 


\section{MÉTODO}

El diseño experimental consiste en un estudio no experimental, exploratorio y transversal. La muestra se realizó con una selección no aleatoria de 100 estudiantes $(\mathrm{M}=20.16 \mathrm{DE}=3.1$ edad y $\mathrm{M}=7$ '291.19 DE = 261.18 ingreso mensual) en una universidad pública en el Estado de México. El 41\% son mujeres y el 69\% son hombres.

Se adaptó la Escala de Percepción del Riesgo de Carreón (2019) que mide expectativas de inconmensurabilidad (vg. "El aislamiento de infectados refleja la propagación del covid-19"), impredecibilidad (vg. "Habrá efectos colaterales desconocidos por la propagación del covid-19") e incontrolabilidad (vg. "El financiamiento de las vacunas contra el covid-19 superará cualquier PIB") con 21 reactivos, considerando cinco opciones de respuesta que van desde $0=$ "nada probable" hasta 5 = "Bastante probable".

Se utilizó la técnica Delphi para establecer la homogeneidad de los conceptos en los reactivos. Encuestamos a los estudiantes en el vestíbulo de la biblioteca de su universidad. Los datos se procesaron con el Paquete de Análisis Estadístico para Ciencias Sociales (SPSS por sus siglas en inglés) y el Análisis Estructural de Momentos (AMOS por sus siglas en inglés). 18,0 versiones. Se estimó la confiabilidad con el alfa de Cronbach, la prueba de validez de Bartlett, KMO y el peso factorial.

El alfa de Cronbach se estimó para establecer la consistencia interna de la escala general y las subescalas. El parámetro Bootstrap se calculó para establecer el muestreo cuando no es posible utilizar completamente los datos y solo se utiliza una parte de la distribución. Se calculó la adecuación y la esfericidad con los parámetros de Kayser Meyer Olkin y la prueba de Bartlett. Se realizó un análisis factorial exploratorio con rotación de promax de ejes principales y criterio de oblicuidad. La prueba de la hipótesis se realizó con la estimación de los parámetros de ajuste y residual.

\section{RESULTADOS}

La Tabla 1 muestra los valores descriptivos del instrumento en el que es posible advertir la distribución normal, fiabilidad y validez del instrumento considerando un umbral de valores entre ,700 y ,900 para la consistencia interna, entre ,300 y ,900 para la convergencia de los factores en un factor de segundo orden común. 
Tabla 1. Descriptivos del instrumento

\begin{tabular}{|c|c|c|c|c|c|c|}
\hline & $\stackrel{\frac{\pi}{3}}{\stackrel{d}{d}}$ & 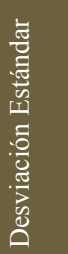 & 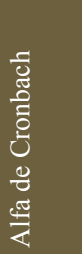 & 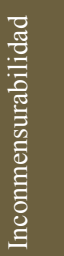 & 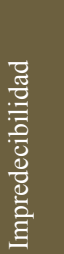 & 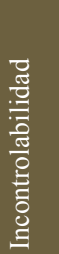 \\
\hline \multicolumn{7}{|l|}{ Subescala de inconmensurabilidad percibida } \\
\hline $\begin{array}{l}\text { El aislamiento de infectados refleja el impacto de la propagación } \\
\text { del covid-19 }\end{array}$ & 4,31 & 1,01 & 0,712 & 0,56 & & \\
\hline $\begin{array}{l}\text { Las estanterías vacías reflejan la amenaza de la propagación del } \\
\text { covid-19 }\end{array}$ & 4,20 & 1,02 & 0,782 & 0,57 & & \\
\hline $\begin{array}{l}\text { La cobertura mediática del coronavirus refleja la propagación } \\
\text { del covid-19 }\end{array}$ & 4,18 & 1,72 & 0,703 & 0,50 & & \\
\hline Las muertes por el covid-19 reflejan el peligro del coronavirus & 4,12 & 1,16 & 0,761 & 0,62 & & \\
\hline $\begin{array}{l}\text { Las compras de pánico reflejan la magnitud del coronavirus } \\
\text { covid-19 }\end{array}$ & 4,08 & 1,03 & 0,752 & 0,53 & & \\
\hline El teletrabajo refleja la magnitud de la propagación del covid-19 & 4,71 & 1,08 & 0,705 & 0,61 & & \\
\hline $\begin{array}{l}\text { Los decretos presidenciales reflejan la importancia de la propa- } \\
\text { gación del covid-19 }\end{array}$ & 4,05 & 1,20 & 0,715 & 0,59 & & \\
\hline \multicolumn{7}{|l|}{ Subescala de impredecibilidad percibida } \\
\hline $\begin{array}{l}\text { Habrá efectos colaterales desconocidos por la propagación del } \\
\text { covid-19 }\end{array}$ & 3,01 & 0,98 & 0,762 & & 0,67 & \\
\hline $\begin{array}{l}\text { Habrá conflictos sociales inéditos por la propagación del } \\
\text { covid-19 }\end{array}$ & 3,28 & 0,92 & 0,710 & & 0,66 & \\
\hline $\begin{array}{l}\text { Habrá crisis económicas inverosímiles por la propagación del } \\
\text { covid-19 }\end{array}$ & 3,17 & 0,93 & 0,751 & & 0,62 & \\
\hline Habrá derrocamientos políticos por la propagación del covid-19 & 3,27 & 0,91 & 0,763 & & 0,50 & \\
\hline Habrá muertes inusitadas por la propagación del covid-19 & 3,82 & 0,95 & 0,770 & & 0,56 & \\
\hline Habrá pánico social inexorable por la propagación del covid-19 & 3,04 & 0,90 & 0,756 & & 0,54 & \\
\hline Habrá desempleo masivo por la propagación del covid-19 & 3,14 & 0,97 & 0,790 & & 0,52 & \\
\hline \multicolumn{7}{|l|}{ Subescala de incontrolobilidad percibida } \\
\hline $\begin{array}{l}\text { El financiamiento de las vacunas contra el covid-19 superará } \\
\text { cualquier PIB }\end{array}$ & 2,56 & 0,32 & 0,761 & & & 0,62 \\
\hline
\end{tabular}




\begin{tabular}{|c|c|c|c|c|c|c|}
\hline & $\stackrel{\frac{\pi}{3}}{\stackrel{0}{\infty}}$ & 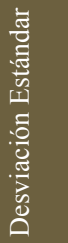 & 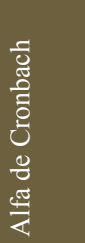 & 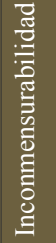 & 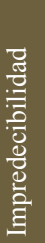 & 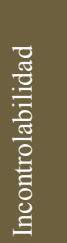 \\
\hline Habrá insuficientes vacunas contra el coronavirus covid-19 & 2,92 & 0,30 & 0,762 & & & 0,53 \\
\hline Las muertes por el coronavirus covid-19 serán interminables & 2,30 & 0,31 & 0,769 & & & 0,49 \\
\hline $\begin{array}{l}\text { El desabasto será exacerbado aún después de una vacuna contra } \\
\text { el covid-19 }\end{array}$ & 2,54 & 0,33 & 0,752 & & & 0,52 \\
\hline $\begin{array}{l}\text { El desempleo se incrementará aún después de la contención del } \\
\text { covid-19 }\end{array}$ & 2,27 & 0,36 & 0,780 & & & 0,69 \\
\hline $\begin{array}{l}\text { Aún después de la erradicación del covid-19 las crisis económi- } \\
\text { cas continuarán }\end{array}$ & 2,27 & 0,35 & 0,761 & & & 0,65 \\
\hline $\begin{array}{l}\text { El pánico continuará, aunque el covid-19 sólo afecte a adultos } \\
\text { mayores }\end{array}$ & 2,59 & 0,39 & 0,761 & & & 0,54 \\
\hline
\end{tabular}

Nota: Elaborada con los datos del estudio. Método de extracción: Ejes principales, Rotación: Promax; Adecuaciòn y Esfericidad $\chi 2==334,12(25 \mathrm{gl}) \mathrm{p}<, 01 ; \mathrm{KMO}=0,764$. Inconmensurabilidad ( $22 \%$ de la varianza total explicada y alfa de 0,792$)$, Impredecibilidad (19\% de la varianza total explicada y alfa de 0,709$)$, Incontrolabilidad (175 de la varianza total explicada y alfa de 0,785$)$. Todos los ítems tienen opciones de respuesta que van desde $0=$ "nada probable" hasta $5=$ "bastante probable".

La consistencia interna, de acuerdo con la recolección y análisis de datos, en la escala general (0.793); Adecuación (KMO =, 764), Esfericidad $\lceil\chi 2=334,1$ (25gl) $\mathrm{p}<, 01\rfloor$ Método: Formas principales, Rotación: Promax. F1 = Inconmensurabilidad (alfa de 0.792 y 22\% de la varianza total explicada); F2 = Impredecibilidad (alfa de 0.709 y $19 \%$ de la varianza explicada); F3 = Incontrolabilidad (alfa de 0.785 y 17\% de la varianza explicada)

Los valores de los parámetros de ajuste y residuales $\lceil\chi 2=135.34(32 \mathrm{gl}) \mathrm{p}=0.054$; GFI $=$ 0,995; CFI = 0.990; RMSEA $=0,003$ J sugiere el no rechazo de la hipótesis nula en relación con las diferencias significativas entre las relaciones teóricas establecidas en la literatura con respecto a las relaciones empíricas encontradas en el estudio. 


\section{DISCUSIÓN}

El aporte del presente trabajo al estado del conocimiento, radica en la exploración dimensional de la percepción del riesgo. Se trata de tres factores relativos al reconocimiento de los límites institucionales, científicos y tecnológicos del Estado con respecto a la propagación del coronavirus covid-19.

En relación a los estudios relativos a los efectos sociales y psicológicos del confinamiento, infección, enfermedad o muerte por el nuevo coronavirus SARS-COV-2 y la enfermedad derivada COVID-19, Hernández (2020) advierte que las tres dimensiones establecidas en el presente trabajo pueden materializarse en una crisis sanitaria en virtud de que los decesos se concentran en espacios cerrados y con densidad poblacional como asilos y hospitales, pero con amplia extensión a zonas de contención de migrantes. Las investigaciones relativas a los efectos en los flujos migratorios y sus diferencias con grupos oriundos permitirán advertir zonas de riesgo en función del tipo de trabajo y la calidad de vida, factores determinantes del contagio, enfermedad y muerte por neumonías asociadas a la pandemia.

Sin embargo, Pérez (2020) observó disposiciones favorables a la difusión del nuevo coronavirus SARS-COV-2 y la enfermedad COVID19 las cuales reducían la ansiedad y la depresión en la medida en que se difundían hallazgos en cuanto a tratamiento o vacunas, pero con un efecto contrario respecto a las noticias de propagación, infección, enfermedad y muerte que en el presente trabajo fueron consideradas como percepciones de riesgos en su dimensión de Incontrolabilidad. Líneas de investigación relativas a esta dimensión permitirán contrastar las fuentes de datos para develar las amenazas percibidas ante la exposición a noticias.

La distinción entre las percepciones de riesgo y utilidad, así como de las actitudes desfavorables o favorables fue de suma importancia para el estudio de Anguiano (2020) quien identificó dos fronteras de conocimiento en una revisión sistemática sobre el instrumento de emprendimiento local. Los efectos esperados de la pandemia sobre la calidad de vida y el bienestar subjetivo destacan las dimensiones de inconmensurabilidad, impredecibilidad e Incontrolabilidad ya que la literatura revisada coincide en áreas de oportunidad para los trabajos mediados por tecnologías de información y comunicación. Líneas de investigación en torno a la utilidad de dispositivos y redes electrónicas no sólo difusoras de información sino productoras de conocimiento facilitarán la observación de la brecha digital entre las generaciones y sectores sociales 
La percepción del riesgo como un proceso híbrido emocional y racional cobra importancia ante un evento incierto cuya propagación mediática incide en la desconfianza ciudadana ante sus autoridades e instituciones de salud. La información difundida en los medios con respecto a los casos de infectados o muertos por el coronavirus covid-19 se estructura en tres dimensiones de percepciones y expectativas ante la acción gubernamental, científica y tecnológica para mitigar o frenar la propagación de la enfermedad.

Esa estructura observable en los eventos de riesgo, subyacentes a emociones y antecedentes de la desconfianza ciudadana ante sus autoridades políticas e institucionales, explicó el 58\% de la varianza total, pero la inclusión de las emociones incrementaría ese porcentaje explicativo.

Los estudios emocionales del riesgo han demostrado que el enojo, el miedo, la zozobra o la indignación son transitorios a los eventos de riesgo, aunque en el caso de la propagación de enfermedades coexisten con la percepción y expectativas de riesgo. La estructura de opiniones ante una agenda sesgada de información en contra o a favor del aislamiento de personas, el financiamiento de vacunas o la mitigación de interrelaciones orienta el aprendizaje de la desesperanza en la sociedad.

Líneas de investigación concernientes a la adhesión al tratamiento, las consecuencias postraumáticas de la enfermedad y los efectos colaterales económicos explicarán el proceso del fenómeno desde el inicio de su propagación, la cúspide de los casos de infectados y la vacuna contra el coronavirus permitirán explicar la estructura racional y emocional de las víctimas como audiencias.

\section{CONCLUSIÓN}

El objetivo del presente trabajo fue contrastar la hipótesis relativa a las tres dimensiones de la percepción del riesgo ante la propagación del coronavirus covid-19, aunque el diseño de la investigación limitó los resultados a la muestra de estudio, sugiriendo la extensión del trabajo hacia la relación entre la estructura perceptual de riesgo con respecto a la estructura emocional que subyace antes, durante y después del evento de riesgo.

En relación con los instrumentos empleados para medir la percepción del riesgo, el presente trabajo corrobora la estructura tridimensional concerniente a la medición, predicción y control de la propagación de la enfermedad, pero sugiere el incremento de la varianza explicada con base en la ponderación de las emociones subyacentes e inherentes. 
Respecto a la fiabilidad y validez del instrumento se reconoce el limite de resultados con respecto a la muestra, pero se recomienda el contraste del modelo de tres dimensiones a fin de abonar al estudio del fenómeno; destacando el nivel de incertidumbre e información de la propagación del covid-19.

En cuanto a las líneas de investigación que se desprenden de los resultados corroborados, se recomienda el seguimiento del fenómeno y su ponderación con instrumentos que recaben las emociones y las conductas antes, durante y después de la propagación a fin de poder explicar el proceso integral del fenómeno.

Las políticas de aislamiento o mitigación empleadas por los gobiernos para contener o minimizar los focos de infección y contagio pueden tomar en cuenta umbrales de desconfianza o fiabilidad ciudadana ante sus acciones y desempeño. Por consiguiente, la edificación de una agenda pública en materia de prevención o mitigación de riesgos supone el concurso de actores políticos y sociales, sectores públicos y privados ante eventos inciertos como el coronavirus covid-19.

\section{REFERENCIAS}

I Aldana, W. (2018). Especificación de un modelo para el estudio de la agenda de la seguridad pública. Atlante, 9(1). $1-20$

I Anguiano, F. (2020). Metanalysis of the effects of entrepreneurship on local development: Implications for the COVID19 coronavirus pandemic. International Journal of Research in Engineering \& Science, 8 (9), 40-47

I Bustos, J. M. (2018). Contrastación de un modelo de decisión prospectiva e implicaciones para una gobernanza universitaria de la sustentabilidad. Margen, 89 (1), 1-16

I Carreón, J. (2018). Confiabilidad y validez de un modelo de gobernanza percibida de la inseguridad. Sin Frontera, 11 (27), 1-53

\Carreón, J. (2017). Una revisión teórica para el estudio de la gobernanza de la seguridad pública. Epsys, 4 (1), $1-15$

I García, C. (2016). Gobernanza del terror a la delincuencia. Eureka, 13 (2), 168-185

I Garcia, C. (2019). Specification of a self-care model. Lux Medica, 42 (1), 15-25 
I Garcia, C. (2020). Specification a model for study of occupational health. Global Journal of Management \& Business Research, 20 (1), 1-6

I García, C. (2017). Gobernanza de la seguridad pública. Revisión de la literatura para una discusión del estado del conocimiento de la identidad sociopolítica delictiva. Margen, 84 (1), 1-17

I Hernandez, J. (2020). Specification of a social intervention model against covid-19. Biomedical Journal of Scientific \& Technical Research, 26 (4), 1-4

$\checkmark$ Hernandez, J. (2020). Specification of a social intervention model against COVI-19. Biomedical Journal of Scientific \& Technical Research, 26 (3), 62-66

I Juarez, M. (2020). Specification a model for study of corporate assistance. Global Journal of Archeology \& Anthropology, 11 (2), 50-54

I Juárez, M. (2017). Fiabilidad y validez de un instrumento que mide dimensiones de seguridad y percepción de riesgo en estudiante de una universidad pública. Revista Internacional de Avances en Ciencias Sociales y Humanidades, 11 (12), 23-13

I Limon, G. A. (2020). Specification a model of need for information about covid-19 coronavirus. International Journal of Social Science Studies, 8 (6), 1-5

I Martínez, E. (2018). La gobernanza de las obras sociales anuncia una violencia en la red. Social Science Learning Educational Journal, 6 (1). 1-3

I Mejía, S. (2016). Efectos psicológicos e violencia e inseguridad en adultos mayores. Eureka, 13 (1), 39-55

I Mendoza, D. (2017). Especificación de un modelo de representaciones propagandísticas en adultos mayores ante la seguridad pública. Tlatemoani, 25 (1), 21-31

I Organización Mundial de la Salud (2020). Estadísticas del coronavirus SARS-COV-2 y la enfermedad COVID-19. Ginebra: OMS

I Organización Panamericana de la Salud (2020). Estadísticas del coronavirus SARS-COV-2 y la enfermedad COVID-19. Washington: OPS

I Perez, G. (2020). Attitudes towards the media spread of the COVID19 coronavirus. International Journal of Humanities \& Social Science Invention, 9 (8), 20-25

I Quintero, M. L. (2019). Reflective factor structure of occupational health governance. Summa, 1 (1), 1-18 
$\checkmark$ Quintero, M. L. (2017). Modelo de expectativas en torno a la seguridad pública en microempresarios del centro de México. Sin Frontera, 10 (26), 1-20

I Rincón, R. M. (2018). Interpretación de discursos en torno al hábito de movilidad para revelar el significado del transporte público. Margen, 90 (1), 1-13

I Secretaria de Salud del Gobierno de México (2020). Estadísticas del coronavirus SARS-COV-2 y la enfermedad COVID-19. SSA

Envío a dictamen: 11 de marzo de 2020

Reenvío: 28 de abril de 2020

Aprobación: 12 de mayo de 2020

Margarita Juárez Nájera. Doctora en Ciencias Sociales, Universidad de Rotterdam, Holanda. Profesora Investigadora, Universidad Autónoma Metropolitana, unidad Azcapotzalco, Departamento de Ingeniería en Energía. Línea de investigación en Educación para la Salud Ambiental. Correo: mjn@azc.uam.mx

José Marcos Bustos Aguayo. Doctor en Psicología, Universidad Nacional Autónoma de México, Profesor de Carrera Titular "C", Facultad de Estudios Superiores Zaragoza, Adscrito al Sistema Nacional de Investigadores, nivel I, línea de investigación en Comportamiento para la Sustentabilidad. Correo: marcos.bustos@unam.mx

Javier Carreón Guillén. Doctor en Administración, Universidad Nacional Autónoma de México, Profesor de Carrera Titular "C", Escuela Nacional de Trabajo Social, Línea de investigación en Gobernanza de la Salud Pública. Adscrito al Sistema Nacional de Investigadores, nivel I. Correo: javierg@unam.mx

Cruz García Lirios. Posdoctorante en Ciencias de la Complejidad, Profesor de Asignatura, Universidad Autónoma del Estado de México, Unidad Académica Profesional Huehuetoca. Línea de investigación en Salud Ocupacional. Correo: chgarcial213@profesor.uaemex.mx 